\title{
Optimal Multicast Routing with Quality of Service Constraints
}

\author{
Vachaspathi P. Kompella, ${ }^{1}$ Joseph C. Pasquale, ${ }^{2}$ and George C. Polyzos ${ }^{3}$
}

\begin{abstract}
We consider the problem of optimal multicast routing with Quality of Service constraints motivated by the requirements of interactive continuous media communication, e.g., real-time teleconferencing. We concentrate on distributed algorithms for determining a tree over the network topology, rooted at the source and spanning the intended destinations. Quality of Service requirements for interactive continuous media typically impose constraints on some metric over the individual paths from the source to each destination, usually in the form of an upper bound on the delay. Thus, we focus on the problem of minimizing the cost of the tree while at the same time satisfying a common constraint over individual source-destination paths. We have shown that this problem is intractable, but have also devised centralized polynomial time heuristics that perform well. Here we present distributed algorithms to minimize tree cost while satisfying the constraints on the paths from the source to each destination.
\end{abstract}

KEY WORDS: Multipoint routing; multimedia communications; interactive continuous media; Steiner tree.

\section{INTRODUCTION}

One of the ways communication can be characterized is by the number of receivers targeted by a sender. Traditional communication modes have been one-to-one, or unicast, and one-to-all, or broadcast. The generalization of these two extremes is multicasting, the simultaneous communication with a select group of recipients. While in the past multicasting has been viewed as a service

\footnotetext{
'IBM Corp., Networking Systems Laboratory, P.O. Box 12195, Research Triangle Park, North Carolina, 27709. (E-mail: vkompella@vnet.ibm.com.)

${ }^{2}$ Computer Systems Laboratory, Department of Computer Science and Engineering, University of California, San Diego, La Jolla, California 92093-0014. (E-mail: pasquale@cs.ucsd.edu.)

${ }^{3}$ Computer Systems Laboratory, Department of Computer Science and Engineering, University of California, San Diego, La Jolla, Califomia 92093-0114. (E-mail: polyzos@cs.ucsd.edu.)
} 
of limited use, often provided as an afterthought, this can no longer be the case. Multicasting is now being viewed as a very important service in networks, especially because multimedia applications can use it effectively. Multimedia is the collection of multiple forms of information presentation, such as text and graphics (classified as discrete media) and audio and video (classified as continuous media). Sound and moving images are a natural form of communication between humans, making the support of continuous media in computer systems an important issue.

It is only recently that technology has become available for computers to be economically equipped with audio and video devices. As these devices proliferate and become standard equipment in personal computers and workstations we expect multimedia group applications to become standard tools for communication and collaboration. A canonical example of such applications is video-conferencing [1, 2]. Furthermore, a major market for Broadband Integrated Services Digital Networks (B-ISDN) is expected to be selective video distribution $[3,4]$, analogous to cable TV, but where the relatively small number of active receivers per surce and the large number of channels (sources) make broadcast solutions impractical.

Due to the very high data volumes of video (and to a much lesser extent audio) when compared with traditional media such as text and graphics, it is important to provide efficient network mechanisms even when high-speed networks are available. The high data rates make resource considerations important despite the decreasing costs of memory and transmission bandwidth. This is particularly important for multipoint applications which, if not designed with optimization in mind, can easily multiply the cost at least by the number of destinations. Although efficient multicasting has been recognized to be a very desirable service $[5,6]$, there is still insufficient support for it in most existing network implementations.

In addition to very high throughput requirements, audio and video are usually associated with real-time interactive applications and thus present rather stringent delay requirements. More precisely, in order for audio and video to be effectively used in interactive communication, i.e., without forcing the communicating subjects to modify their behavior from that of face-to-face communication, the delay between transmitter and receiver is expected to be upper bounded. Various guidelines set this tolerance between 40 and $600 \mathrm{~ms}[7,8]$, depending on the particular application. Sometimes however, different metrics are targeted to be bounded (on an end-to-end basis), e.g., delay jitter [9].

Multicasting issues have attracted considerable attention recently. Surveys on multicasting for distributed systems can be found in Frank et al. [10], and Ahamad (Editor) [11]. Much work has been done in the context of IP multicasting for the Internet (e.g., [12-15]); however, this is not our primary focus here. There is also significant on-going work in the area of high-speed switch 
design, particularly for Asynchronous Transfer Mode (ATM) switches, to efficiently support multicasting in the nodes of the network [16-18]. The deployment of such switches is a prerequisite for the approach and techniques described here. Finally, a related problem to the one we consider here has been addressed by Verma and Gopal [19]. The authors develop heuristics for finding the capacity-constrained minimum cost tree which minimizes the cost of bandwidth reserved for point-to-point communication from destinations to the source and the number of extra nodes in the tree which increase the cost of bandwidth reserved from the source to the destination.

In this paper, we discuss the problem of multicast routing with Quality of Service (QoS) constraints motivated by the requirements of multipoint multimedia communications (specifically, interactive continuous media communication). Briefly, the relevant characteristics of this problem are: (1) data must be sent to multiple destinations, (2) the volume of the data is large, requiring high bandwidths, and (3) the value of the data is sensitive to delay; if it is received beyond a threshold dictated by human perception, it becomes useless. Since the volume of the data is large, minimizing duplication of transmission is important. And since the data is sensitive to delay, finding short routes which can transport data concurrently to the multiple destinations is important.

The remainder of this paper is organized as follows. In Section 2, we discuss some central issues in multicast routing for interactive continuous media. In Section 3, we focus on the static, one-to-many version of the problem and formulate a graph theoretic problem to optimize multicasting under QoS constraints. In Section 4, we present distributed heuristics for the problem and in Section 5 we provide an empirical performance evaluation of the heuristics. Finally, conclusions are presented in Section 6.

\section{ISSUES IN MULTICAST ROUTING FOR INTERACTIVE CONTINUOUS MEDIA}

Multicasting uses trees over the network topology for transmission. There are two reasons for basing multicast routes on trees: (1) the data can be transmitted in "parallel" to the various destinations along the branches of the tree, and (2) a minimum number of copies of the data are transmitted, with duplication of data being necessary only at forks in the tree. There are several issues that present themselves in multicast tree construction, particularly for continuous media and multimedia communication, which we discuss next.

For continuous media traffic QoS considerations are critical and thus resource allocation and reservation issues are important. Therefore, it is now generally accepted that some form of connections, possibly with some level of resource reservations, are needed to best support continuous media applications. For connection-oriented communication, where all traffic follows the selected 
path(s) (at least until a reconfiguration), the time required to make an optimal decision can more easily be justified because of the (assumed) duration of the connection and the large amount of traffic using the route, and thus the consequences of a sub-optimal choice.

Routing decisions, and optimization in particular, are based on the network parameters, e.g., cost and performance of links and nodes. However, these change with variations in the traffic and thus must be appropriately updated. Therefore, the routing problem is dynamic. Nevertheless, because of its complexity, static or quasi-static versions are usually considered. We can obtain a quasi-static solution to the routing problem using the static solutions and updating the weights of the graph.

In the specific case we consider here we associate two metrics with each link in the network: a cost and a delay. These two metrics can play more general roles; in particular, the "delay" metric could be used to represent and satisfy various other constraints on each source-destination path. However, the more direct use of the metrics is for cost to represent the actual cost of using the link for the distribution of the information to the multiple destinations, and for the delay to represent the actual delay introduced by the link. To simplify obtaining and using network status information we can use proxies for these metrics.

Assuming static capacity allocation, and costs and delays that are independent of network load, a quasi-static solution can be obtained as follows. We start with a graph representing the network topology with the specific link capacities and link costs and delays. We assume that routing decisions are made sequentially. Each time a routing decision is made, the available capacity in the graph is updated by subtracting from the capacity of each link used in the tree the capacity of the connection being routed. A graph for each routing decision is obtained from the updated network graph by removing edges that cannot provide the capacity required by the connection under consideration. Since we assumed costs and delays independent of network load (i.e., we concentrated on propagation, transmission, and processing delays and neglected queueing delays), no updating is required on these metrics of the links. In this way, each specific routing decision is made using a static approach. However, the process takes into consideration resources consumed by established connections.

One important issue to address is how many trees to use for a multicast. If there are multiple sources, e.g., in a video-conference, then using one tree for the entire conference is certainly a desirable solution, in terms of network state information maintained. This is the main argument of the Core Based Tree (CBT) approach to Internet multicasting [15]. The CBT approach uses one tree to deliver packets from all sources to all destinations in the same group. Compared to source-rooted trees (e.g., Deering and Cheriton [12]), it decreases the amount of routing information which needs to be maintained by each network node and communicated to other nodes. However, with respect to economy and 
QoS support, the single tree approach suffers. In fact, there may not be a single tree that can be used by the conference, or the QoS provided by the tree may be severely compromised. Furthermore, the links of such a tree would have to assume good properties in both directions.

The alternative is to use a distinct tree for each source. Individual trees can lead to optimal solutions that minimize total cost and satisfy QoS constraints, such as an end-to-end delay tolerance, without compromises. However, the amount of state information needed to be maintained is high. In addition, reconfiguration (when changes occur either to network topology or in group membership) could take a long time to complete while state information is being updated. Hierarchical multicast, a compromise between these two extremes, is proposed by Yen and Akyildiz [20].

When multiple continuous streams are considered, e.g., in the case of a teleconference with multiple audio, video, and textual streams emanating from each source, the problem is compounded. This problem is worth considering because the QoS requirements of different media are typically different, and the QoS requirements even for the same medium type could be differently specified from source to source. Thus, optimality would suggest that each multimedia stream be allocated its own tree, satisfying its QoS specification. For example, in an ATM network, such a solution would lead to multiple multipoint virtual circuits from each group member (source), one for each particular medium stream.

\section{MULTICAST TREE SELECTION}

In this section we concentrate on the static problem of multicasting from a single source to multiple destinations. In the context of our previous discussion for group communication, this implies that multiple independent trees will have to be selected and maintained for group communication (from all to all). We first discuss the various goals that can be set and then formulate a specific optimization problem.

Algorithms for constructing multicast trees have been developed with different optimization goals in mind. Typical goals are: (1) to minimize some delay metric, e.g., mean path delay from the source to the destinations, or (2) to minimize the total cost of the tree. For point-to-point (unicast) communication the different goals of minimizing cost or minimizing delay lead to the same solution algorithms, i.e., shortest path algorithms. The only difference is in the metrics used, i.e., link cost or delay; sometimes in real networks even ad hoc metrics, not representing either cost or delay directly, are used. In the multipoint case, however, the two goals are distinct and lead to different problems.

A minimum path delay tree can be constructed in $O\left(n^{2}\right)$ time using Dijkstra's shortest path algorithm [21] (and pruning off the branches of the tree that 
do not contain any of the destination nodes), where $n$ is the number of nodes in the graph. On the other hand, the least cost tree is called a Steiner tree [22], and the problem of finding a Steiner tree is known to be NP-complete [23]. Several (centralized) algorithms that construct low-cost multicast routes [2427] are based on heuristics for approximate Steiner trees [28-30]. Empirical observations show that the heuristics produce near optimal trees quickly. These algorithms take polynomial time, ranging from $O\left(n^{3}\right)$ to $O\left(n^{4}\right)$. Furthermore, many of the algorithms are guaranteed to provide solutions within a constant factor of cost from the optimal.

In our formulation, a multicast tree is a constrained Steiner tree, i.e., a delay-bounded minimum cost tree, where the delay bound is specified by the application performing the multicast. We formulate this precisely next.

\subsection{The Constrained Steiner Tree}

The constrained Steiner tree (CST) problem can be formulated as follows. Given a graph $G=(V, E)$ with node set $V$ and edge set $E$, we define the following weight functions on the edges:

$$
\mathcal{C}: E \rightarrow R^{+} \text {, a positive real edge cost function }
$$

and

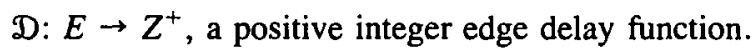

On this graph, we designate a source node $s$ and a set of destination nodes $S$, called the multicast group. Given a delay tolerance $\Delta$, a constrained spanning tree $T$ is a tree rooted at $s$, that spans the nodes in $S$, such that for each node $v$ in $S$, the delay on the path from $s$ to $v$ is bounded above by $\Delta$. We can now describe the constrained spanning tree formally as follows: for each $v \in S$, if $P(s, v)$ is the path in $T$ from $s$ to $v$,

$$
\sum_{e \in P(s,(u)} D(e)<\Delta
$$

The constrained Steiner tree can now be described as a constrained spanning tree such that

$$
\sum_{e \in T} \mathfrak{e}(e) \text { is minimized. }
$$

We note that this problem is $N P$-complete, as shown in [31].

We refer earlier to the two metrics as cost and delay because this is the most obvious relationship with the application we focus on. However, the intrinsic requirements for the two metrics is that one (what we call cost) is additive over the (whole) tree, while the other (delay) is additive along individual source-destination paths. Furthermore, while the former is our optimization 
goal, the latter (together with the application specified tolerance) provide a set of constraints that need to be satisfied by the solution. For example, our formulation would directly model the similar problem of minimizing cost under an end-to-end jitter constraint (if one could assume that jitter is given and additive along the source-destination path).

Another interesting problem that is supported by the above formulation is the following. Assume that reliable multicast in a probabilistic sense is the goal and that independent success probabilities are given for each network link (edge). A simple reliability constraint could then be expressed as a lower bound on the success probability along each path. Because of the independence assumption these constraints can also be expressed as an upper bound on the sum of the negatives of the logarithms of the success probabilities along the path. Therefore, the formulation can also be used to minimize the cost of multicasting under some forms of reliability contraints.

To illustrate the various optimization goals and the corresponding solutions obtained, consider the graph shown in Fig. 1. The pair of numbers along each edge represent, respectively, the cost and delay for that edge. The source node is $\mathrm{F}$, and the multicast group is $\{\mathrm{B}, \mathrm{D}, \mathrm{E}, \mathrm{H}\}$. The delay constraint is $\Delta=5$; thus, all paths must have a delay less than or equal to 4 . Figure 2 shows the optimal tree (CST) which has a cost of 20 units (and maximum delay equal to 4 units). In this case this happens also to be a Steiner tree (i.e., the minimum cost tree, irrespective of constraints). Furthermore, notice that another Steiner

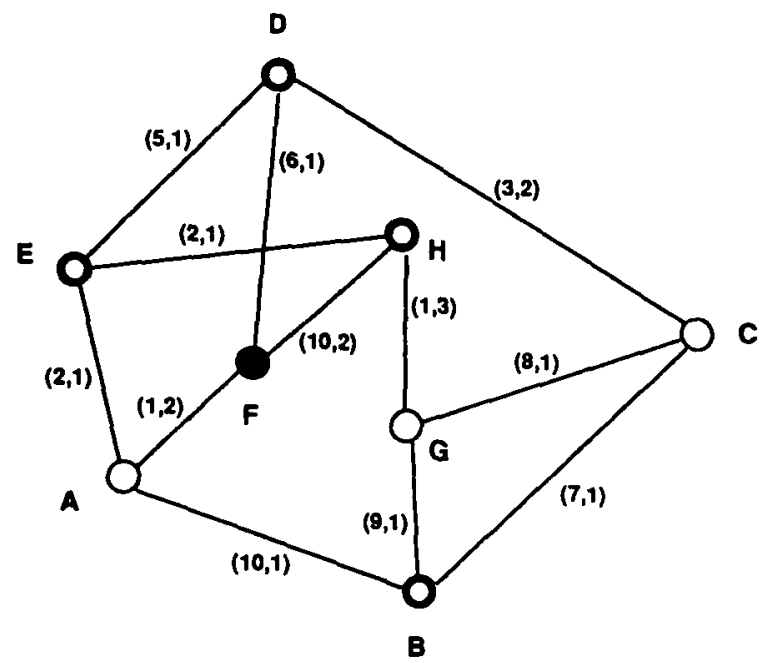

Fig. 1. The graph used in explaining the heuristics. The pair of numbers along each edge represent the cost and delay for that edge, respectively. The source node is $F$, and the multicast group is $\{B$, D, E, H\}. 


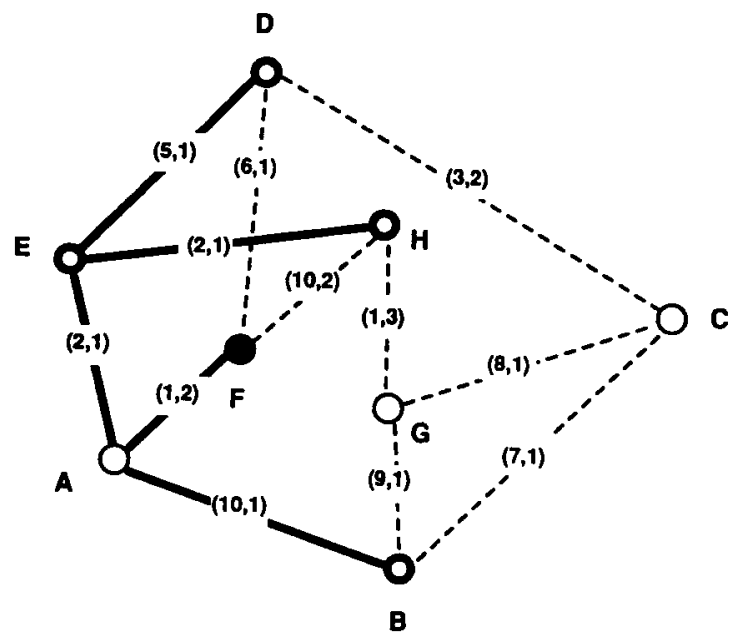

Fig. 2. The optimal constrained Steiner tree on $S=\{\mathrm{B}, \mathrm{D}, \mathrm{E}, \mathrm{H}\}$ with $\Delta=5$ and $s=\mathrm{F}$. The total cost is 20 units and the maximum delay is equal to 4 units.

tree is obtained if we substitute the edges HG and GB instead of the edge AB in the tree shown (i.e., the cost remains equal to 20 units and the multicast group remains covered). On the other hand, this last tree is not a CST anymore since the delay from $\mathrm{F}$ to $\mathrm{B}$ is now equal to 8 units, i.e., more than the delay tolerance.

To continue along these lines, if a shortest path algorithm is run using the delay metric along the edges, we obtain the solution shown in Fig. 3. This

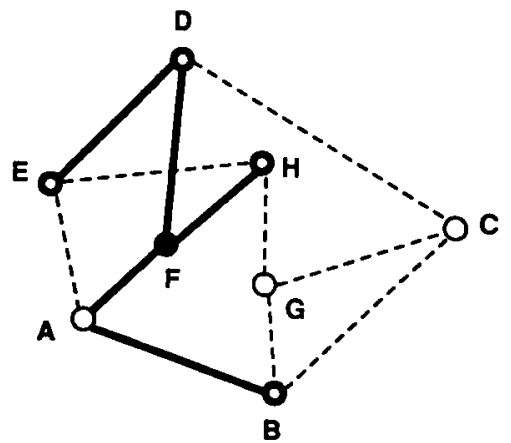

Fig. 3. The tree produced by the shortest path algorithm from the source to the destinations using the delay metric along the edges. The total cost is equal to 32 units and the maximum delay is equal to 3 units. 


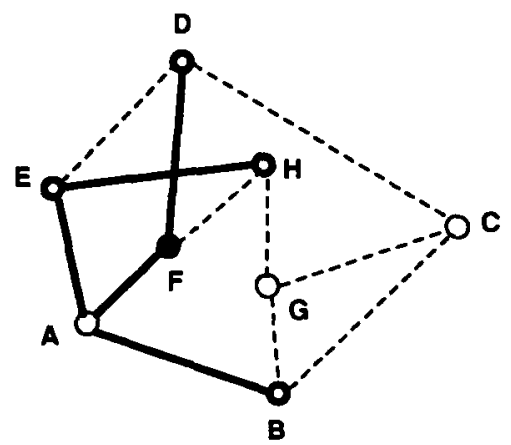

Fig. 4. The tree produced by the shortest path algorithm from the source to the destinations, but using the cost metric along the edges. The total cost is 21 units and the maximum delay is equal to 4 units.

solution, indeed, minimizes the path delays, thus exhibiting a maximum delay of 3 units. On the other hand, its cost is equal to 32 units. Finally, a shortest path algorithm can also use the cost metrics along the edges. In this case we obtain the solution shown in Fig. 4 with a cost equal to 21 units and a maximum delay of 4 units.

We believe that these examples illustrate the basic differences between the two approaches, i.e., the shortest path based solutions and the Steiner tree based solutions. The former are tractable but do not attempt to optimize on the cost of the tree because they have no way of forcing the reuse of paths selected for other destinations. However, when the shortest path algorithm is applied to the cost metrics, the solution might be very good, as is the case with the solution shown in Fig. 4. On the other hand, the Steiner tree based solutions reuse already selected paths in their effort to achieve an overall minimum cost; however, in doing so they are computationally complex. Finally, it is rather clear from the discussion on Fig. 2 that a Steiner tree can impose a long source-destination path, while there could be other low cost (even optimal, i.e., Steiner) trees that impose shorter source-destination paths in the sense of delay.

\subsection{Centralized Routing Algorithms}

We have presented and evaluated centralized heuristics for the solution of the CST problem in [32]. Here we describe them briefly in order to compare them to the distributed heuristics. The basic strategy is to construct a closure graph on the source and destination nodes only. The closure graph encapsulates the costs of the cheapest delay-bounded paths between any two nodes, and the corresponding delays. The cheapest delay-bounded path between two nodes is 
defined as the least cost path between those nodes such that the delay along the path is less than the delay constraint. Then, a spanning tree of this closure graph, rooted at the source, provides an approximate CST. Note that by choosing edges carefully, this centralized algorithm can guarantee a solution, when one exists, which based on experimental results appears to be close to optimal.

We have empirically investigated the performance of this algorithm on randomly generated graphs of up to 100 nodes, with average connectivity of 15 nodes, for group sizes up to 30 nodes, and various delay tolerances. On the average, the trees constructed using this algorithm are within $5-10 \%$ of the optimal cost, and they satisfy the delay requirements [32]. An interesting result of the study is that the shortest delay tree (i.e., the tree of shortest delay paths from the source to the destinations without attempting to optimize on the cost), has a cost that is, on the average, $75 \%$ more expensive than optimal. We have also tried the algorithm on graphs with the T1 NSFNET topology, with similar results. Thus, in terms of cost, the shortest delay tree provides a poor solution.

In dealing with larger networks, where the cost of maintaining consistent information at all nodes regarding the network status may be prohibitive, a distributed approach seems more appropriate. In the next section we present two distributed algorithms using two different heuristics for selecting edges in the tree. These distributed algorithms use only a small amount of local information.

\section{DISTRIBUTED ALGORITHMS FOR THE CONSTRAINED STEINER TREE}

When a network is small, it is possible to maintain a consistent view of the network status by broadcasting this information. As the network gets larger, this process becomes less reliable, less accurate, and more expensive. In this section we consider distributed algorithms that do not need the source to have complete knowledge of the network topology and state; instead they use only information that is passed on by neighboring nodes. Furthermore, as we will see in Section 5, the best distributed heuristic typically comes within $10 \%$ of the cost of the best centralized heuristic.

The first approach, $S P T$, simply constructs the tree of minimum delay paths that connect the source to each destination. This is a distributed version of the Bellman-Ford shortest path algorithm [33]. Obviously, the tree can be pruned to remove unnecessary branches. It is guaranteed to be successful in finding a constrained spanning tree (if one exists), but does not attempt to optimize on the cost of the solution. Figure 3 shows the solution produced by SPT when run on our example graph. As we have seen, this tree has a cost of 32 units.

We now present two distributed algorithms using two heuristics to select 
edges in the tree, $f_{C}$ and $f_{C D}$, which are described in detail later. The general form of the two distributed algorithms is the same, differing only in the criterion they apply to select edges. These two algorithms are based on a distributed minimum spanning tree (MST) algorithm, which essentially mimics Prim's MST algorithm. The reader is referred to Gallagher et al. [34] for details on this distributed MST algorithm.

\subsection{Satisfying the Delay Bounds}

In order to construct an approximate constrained Steiner tree in a distributed way we use the distributed MST algorithm operating on the cost as the edge metric (e.g., see Fig. 4). The most significant problem with using this approach is that there is no way to know a priori whether the tree is growing in the right direction. Since the distributed MST algorithm operating on the cost metric does not place any restrictions on delay, it is possible that a tree grown this way fails to meet the delay bound. To avoid this, one could discard edges that cause a delay bound violation. However, this is not a strong enough condition to guarantee that the distributed MST algorithm will always produce a delay constrained tree when one does exist.

To illustrate this point, consider Fig. 5, an instance where A is the source, $\mathrm{D}$ is the destination and $\Delta=3$, so that the path delay from $\mathrm{A}$ to $\mathrm{D}$ should not exceed 2. If edges were chosen based on cost, then edges $A B$ and $B C$ would be selected over AC because they are cheaper. The algorithm would then terminate declaring no solution exists because selecting edge $C D$ would cause a delay bound violation. However, $\{\mathrm{AC}, \mathrm{CD}\}$ form a solution.

One way to resolve this problem is to choose an edge that causes a cycle and then break the cycle by deleting some edge (or edges) from the tree. In this case, A has very few choices, so the resolution works. However, in general, it is not obvious which edge to add to form the cycle, and which to delete to break the cycle.

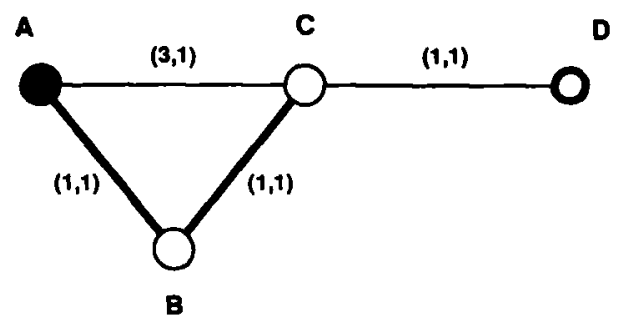

Fig. 5. Example where $D M C T_{C}$ and $D M C T_{C D}$ fail. $A f t e r A B$ and $B C$ have been selected, $D$ cannot be reached. $\Delta=3$. 
The problem with nodes having no delay information other than the adjacent link delays is that this very limited horizon does not permit them to decide whether the direction being explored is delay-bounded or not. This problem can be obviated if each node has available the minimum delay to every other node in the graph (e.g., by running the Bellman-Ford distributed algorithm on the delay metric). Using this approach, the previously incorrect termination can be circumvented in the following way. At the first stage, A would have to decide between edges $A B$ and $A C$. When $B$ responds to a query from $A$ regarding the minimum delay from $B$ to $D$, A can easily conclude that with a delay of 2 from $B$ to $D$, no solution exists that includes $A B$. A can then turn to $C$, and build the correct solution.

We will return to the problem of possible termination without a solution again, as this can happen even with our algorithm. However, this time, we shall demonstrate that there is a convenient procedure to decide how to resolve this problem, by choosing to make and break cycles in such a way that the number of reachable destinations increases. As a consequence, we know that with $k$ destinations, the maximum number of cycles that have to be resolved cannot exceed $k-1$.

\subsection{Overview of the Distributed Algorithms}

We now provide an overview of the operation of the distributed algorithms. The full algorithm is given in Appendix A, with the metrics used for edge selection described in the next sub-section.

Every edge $(v, w)$ is initially marked Unknown. As nodes receive responses to TEST and ADJUST messages, they change the status of edges to Usable, Cycle, or Unusable. A Usable edge is an edge that can potentially be selected because there is at least one unreached destination that can be reached from it without exceeding the delay bound. An Unusable edge is an edge that is not Usable. A Cycle edge is an edge that could potentially be used, but it would create a cycle. Before sending an ADD message for edge $(x, y)$, node $x$ marks $(x, y)$ as Tree to signify that it is a tree edge. On receiving the ADD message, node $y$ marks edge $(y, x)$ as Reverse to mark the reverse path back from $y$ to the source.

The set of unreached destinations $S^{\prime}$ is initially the set of all destinations $S$. As destination nodes are added to the tree, the source, $s$, removes them from $S^{\prime}$ and when necessary reports (in a FIND message) the newest destination node added to the tree. In that way, nodes get to know what $S^{\prime}$ is. Every new node in the tree is communicated the set $S^{\prime}$ in the ADD message. Finally, when the source determines that all the nodes to be covered are in the tree, it can propagate a message up the tree to eliminate unnecessary branches that lead to nodes not in the destination set. 


\subsection{Edge Selection Functions}

The important question to determine here is how to choose between competing edges. Several heuristics can be used to select the best edge to extend the subtree. We have looked at two edge selection functions. Firstly, it could be argued that cost is the over-riding determinant of the route. Thus, a first choice could be based on selecting the cheapest edge out-going from the subtree constructed so far, ensuring that it does not violate the delay bound. We call this selection function $f_{C}$, and the consequent variant of the distributed algorithm $D M C T_{C}$.

$$
f_{C}= \begin{cases}\mathfrak{C}(v, w) & \text { if } \mathbb{P}(v)+\mathfrak{D}(v, w)<\Delta \\ \infty & \text { otherwise }\end{cases}
$$

where

$$
\begin{aligned}
\mathcal{C}(v, w) & =\text { cost of the link }(v, w) \\
\mathcal{D}(v, w) & =\text { delay on the link }(v, w) \\
\mathcal{P}(v) & =\text { delay on the path from } s \text { to } v \text { in the tree. }
\end{aligned}
$$

A second choice could take into account the fact that delay also plays a prominent role. We opted to use a function that modulates the cost of an edge by a factor that biases the choices towards lower delay. Define the residual delay at a node $v$ as $\Delta-P(v)$. Then, it would be desirable to have a large value of residual delay. If the residual delay on a path is large, then the likelihood of reusing that path to reach other destinations improves. This tends to lower the cost of the tree. The function we used was simply the cost of an edge divided by the residual delay of that path. We call this selection function $f_{C D}$, and the corresponding variant of the distributed algorithm $D M C T_{C D}$.

$$
f_{C D}= \begin{cases}\frac{\mathcal{C}(v, w)}{\Delta-[\mathcal{P}(v)+\mathscr{D}(v, w)]} & \text { if } \mathcal{P}(v)+\mathscr{D}(v, w)<\Delta \\ \infty & \text { otherwise }\end{cases}
$$

Figure 6 shows the edges $D M C T_{C}$ uses in constructing a multicast tree. In the first step,.shown in Fig. 6(a), F can choose one of three edges, FA, FD, and FH with $f_{C}$ values 1,6 , and 10 . Thus, it chooses FA. The tree constructed by $D M C T_{C}$ has a cost of 20 . Figure 7 shows an instance of the working of $D M C T_{C D}$. The tree generated by $D M C T_{C D}$ has a cost of 25 . In the first step, shown in Fig. 7(a), F can choose one of three edges, FA, FD, and FH with $f_{C D}$ values $0.3,1.5$, and 3.3 , respectively. Thus, it chooses FA. We note that the 


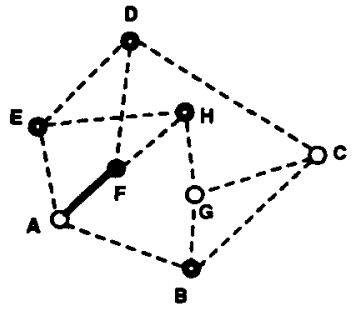

(a)

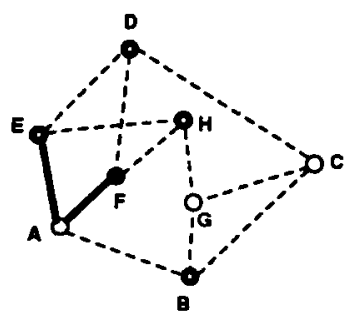

(b)

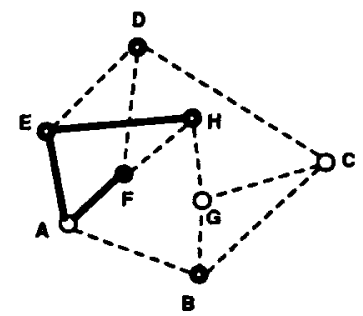

(c)

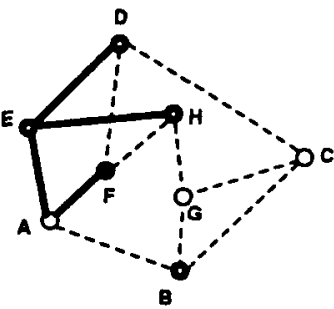

(d)

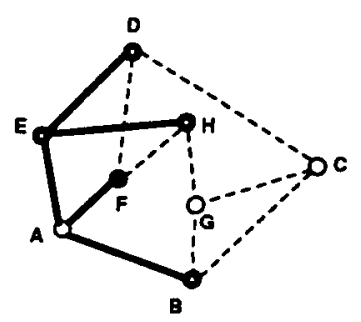

(e)

Fig. 6. (a)-(d) Four stages in producing the tree using $f_{C}$. (e) The final tree produced by $D M C T_{C}$.

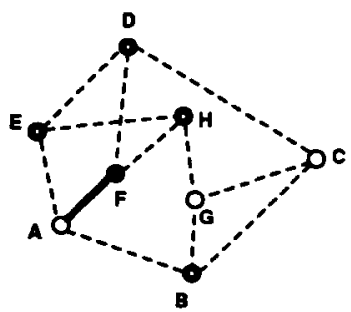

(a)

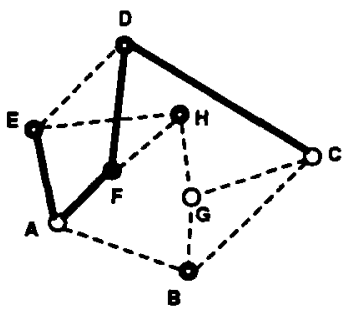

(d)

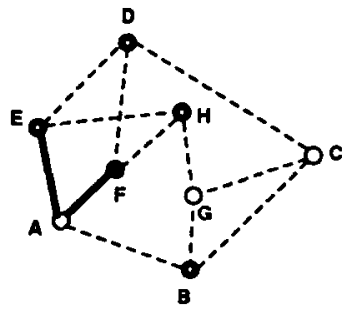

(b)

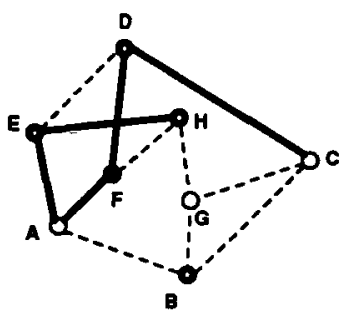

(e)

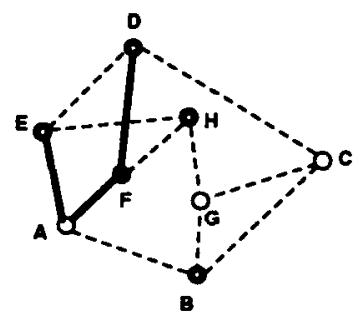

(c)

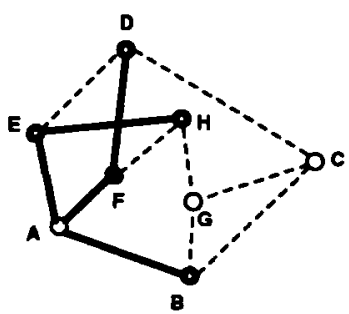

(1)

Fig. 7. (a)-(e) Five stages in producing the tree using $f_{C D}$. (f) The final tree produced by $D M C T_{C D}$. 
algorithm also selects edge DC, however, we prune the tree to remove branches that lead to leaf nodes not in $S$.

\subsection{Eliminating False Termination}

We now address the problem of false termination. It is still possible to run into a situation where the algorithm reports no solution exists, while a solution does exist. Consider for example Fig. 8(a). The source is $A$, the destinations are $\{C, D\}$ with a delay constraint $\Delta=6$. If $f_{C}$ is being used, then the first step would be to choose AU, the second to choose AV, the third to select $U W$, and the fourth to pick WD, to get to the position in Fig. 8(b). Now it is not possible to meet the delay constraint to $C$.

At this point, however, we know what to do. Let the current tree be called $T=\{A U, A V, U W, W D\}$. The source asks that a cycle be created by adding VW. $\mathrm{V}$ and $\mathrm{W}$ will then discover their common ancestor (in this case, $\mathrm{A}$ ) in the tree by propagating messages along their Reverse edges. The common ancestor can then send a request towards $\mathrm{V}$ asking that the cycle be broken by some edge on the path that can be traced backward from W to A (following the edges with status Reverse), i.e. WU. This procedure adds a node to the tree from which it is now possible to reach one of the unreached destinations. By repeating this process a maximum of $n-1$ times, we must end up with a path from the source to an unreached destination, since a path can have at most $n-$ 1 edges. This also relies on the definition of a Cycle edge $(x, y)$, which is an edge with the property that $P(x)+D(x, y)+S D(y, w) \leq \Delta$ for some $w \in S^{\prime}$.

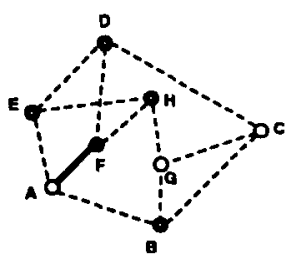

(a)

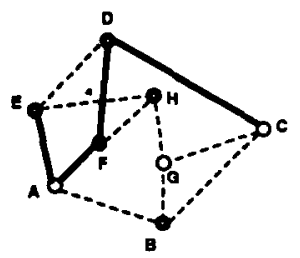

(d)

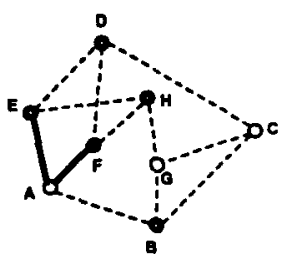

(b)

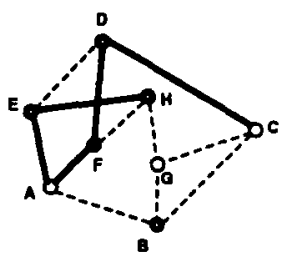

(b)

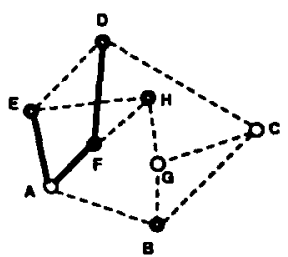

(c)

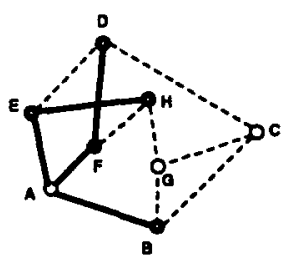

()

Fig. 8. An example using $D M C T_{C}$ with cycle resolution. 
In other words, in the example, $\mathrm{VW}$ is a Cycle edge, so that the path delay at $\mathrm{V}$ plus the delay on edge $\mathrm{VW}$ plus the minimum delay from $\mathrm{W}$ to $\mathrm{C}$ is delaybounded.

While the first edge on the reverse path can be used to break the cycle, it may often turn out to be better to propagate the messages all the way back to the ancestor, so that we pick up as many edges on the path from $\mathrm{V}$ to $\mathrm{C}$. The criterion for choosing which edge to use to break the cycle is given in Appendix B.

The cycle make-and-break procedure will remove edge WU. We now get $T^{\prime}=\{A U, A V, V W, W D\}$. Since the delay on the path from $\mathrm{A}$ to $\mathrm{W}$ in $T^{\prime}$ is smaller than the path from $\mathrm{A}$ to $\mathrm{W}$ in $T$, all the nodes reachable from $\mathrm{W}$ are still reachable in $T^{\prime}$. However, now $\mathrm{C}$ is also reachable. Finally, we prune the unnecessary edge (AU), to yield the tree in Fig. 8(c).

This cycle make-and-break phase can now be included in the algorithm, between steps 6 and 7. It is given in Appendix B.

\subsection{Complexity of the Heuristics}

The distributed algorithms $D M C T_{C}$ and $D M C T_{C D}$ are based on the distributed MST algorithm. However, there are four differences. Firstly, instead of allowing the tree to be built up in fragments that eventually connect together, this tree is built from a single root. This corresponds to the distributed MST algorithm running with a single node woken up initially. This would cause the distributed MST algorithm to run with $O\left(n^{3}\right)$ number of messages [34]. Thus, the number of messages for $D M C T_{C}$ or $D M C T_{C D}$ would be $O\left(n^{3}\right)$ also. A second difference is that in the distributed MST algorithm, the decision on which edge should be added next moves to the endpoints of the new edge added to the tree. In our algorithms, this point of decision is at the source. However, this does not add to the number of messages sent.

Finally, $D M C T_{C}$ and $D M C T_{C D}$ have two more phases. Firstly, the cycle make-and-break phase. The cycle-making phase requires an extra set of CYCLEADD messages to be sent from the source to each node in the tree, a MAKE-ANDBREAK message, and a CYCLE-BREAK message. These can total at most $O(n)$ for each cycle breaking. This is because there can be at most $n-1$ edges in a tree. As noted before, each time a cycle is made and broken, we add an edge that takes us closer to an unreached destination. This process of adding an edge may have to be repeated at most $n-1$ times, since there are at most $n-1$ edges in a path. Thus, within $O\left(n^{3}\right)$ messages, the cycle make-and-break phases can be completed, still yielding an $O\left(n^{3}\right)$ algorithm. For comparison, note that for the shortest paths in the centralized case, Dijkstra's shortest path algorithm takes $O\left(n^{2}\right)$ time [21], while the distributed Bellman-Ford algorithm takes $O\left(n^{3}\right)$ time [33]. 


\section{EMPIRICAL PERFORMANCE ANALYSIS}

Because the cost of comparing the heuristics with the optimal is prohibitive, we compared the distributed algorithms against a pseudo-optimal heuristic, $C M C T_{C}$, which is the better of the two centralized heuristics [32], and the best among all the heuristics that we have come up with so far. The metric we used to compare the heuristics is the normalized surcharge with respect to the pseudooptimal, given by:

$$
\hat{\delta}_{H}=\frac{T_{H}-T_{C M C T C}}{T_{C M C T_{C}}}
$$

where

$$
\begin{aligned}
T_{H} & =\text { cost of tree using heuristic } H \\
T_{C M C T_{C}} & =\text { cost of tree using heuristic } C M C T_{C}
\end{aligned}
$$

The heuristics were run on randomly generated graphs with low average degree. The graphs we generated for the evaluation of the heuristics had between 50 and 100 nodes, and an average degree between 5 and 15. The nodes in the graphs were randomly placed in a unit square, and the edge delays were proportional to the Euclidean distance separating the endpoints. We used both unit edge costs and random edge costs generated uniformly from the interval [16, 35]. The random edge costs match typical values for costs used in the $T 1$ NSFNET backbone network. However, earlier results indicate that the properties of the heuristics and their relative performance remain the sarne for both unit costs and random costs. Each experiment generated 100 graphs with identical parameters: number of nodes, maximum degree of each node, delay bound, and size of the multicast group. Each graph was then checked to ensure that a solution existed. The confidence interval for $\delta$ at the $95 \%$ confidence level is about $1-2 \%$ for all the graphs shown.

An interesting feature of the graphs in Fig. 9 is that $D M C T_{C D}$ outperforms $D M C T_{C}$ for large graph sizes. Thus, as the tree size increases, it is apparent that introducing a delay component in the selection function becomes important for the success of the distributed algorithms. However, the same is not true for the centralized algorithms [32]. Since they operate on a closure graph, which has an implicit delay component because of the manner in which it is constructed, it is better to use $f_{C}$ than $f_{C D}$ because there is no unnecessary trading of cost for lower delay.

For large multicast group sizes, the algorithms $C M C T_{C D}, C M C T_{C}$, $D M C T_{C D}$, and $D M C T_{C}$ converge to the minimum spanning tree. This is borne out by Fig. 10, where the deviation from $C M C T_{C}$ is very small. For large delay tolerances, the performance of $D M C T_{C D}$ is only marginally better than that of $D M C T_{C}$, as can be seen from Fig. 11. This is to be expected, by observing that 
Normalized Surcharge $(\hat{\delta})$ vs. Nodes

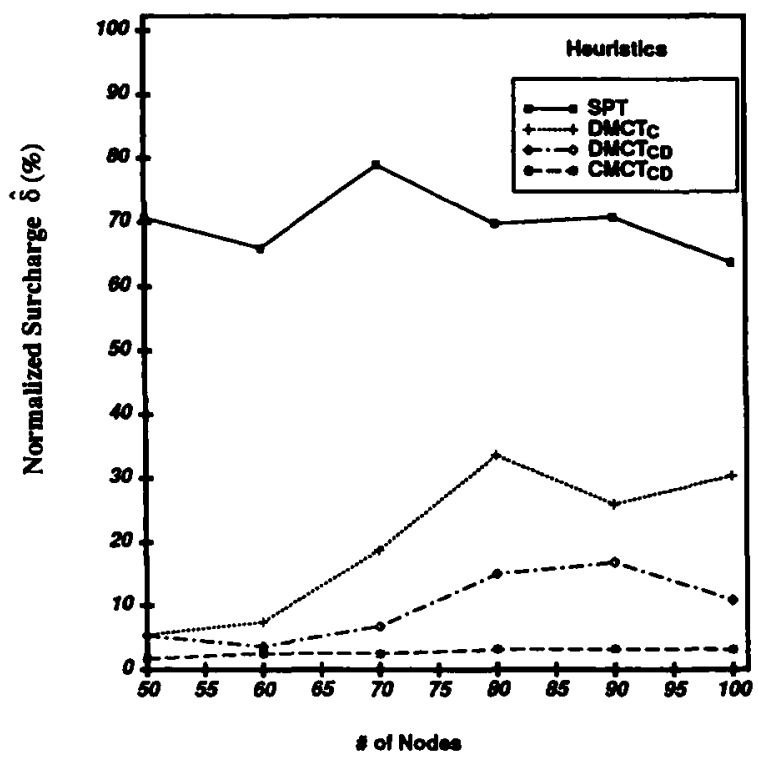

Fig. 9. Normalized surcharge $\hat{\delta}$ vs. number of nodes, for group size $=15, \Delta=80$, and maximum degree $=30$, when compared with $C M C T_{C}$.

$f_{C D}$ behaves like $f_{C}$ when the value of $\Delta$ in the denominator is very large. The same is true for $C M C T_{C D}$ and $C M C T_{C}$.

\section{CONCLUSIONS}

Multipoint multimedia communication is becoming increasingly important. We have discussed the main routing issues for networks supporting multicasting for continuous media and have presented distributed routing algorithms that satisfy some forms of application Quality of Service parameters, typically imposed to achieve acceptable levels of interactivity.

We have formulated the multicast routing problem with QoS constraints as a constrained Steiner tree problem. The objective function is minimal overall cost of the tree with bounded delay paths from source to each destination. We note that the term cost may be replaced by an additive metric for the overall tree optimization goal and the term delay by a path additive constraint. This problem then also applies to VLSI routing, where the goals are minimal overall length of wire used for connecting source and destinations, as well as reducing the number of hops to keep signal propagation delay down. 


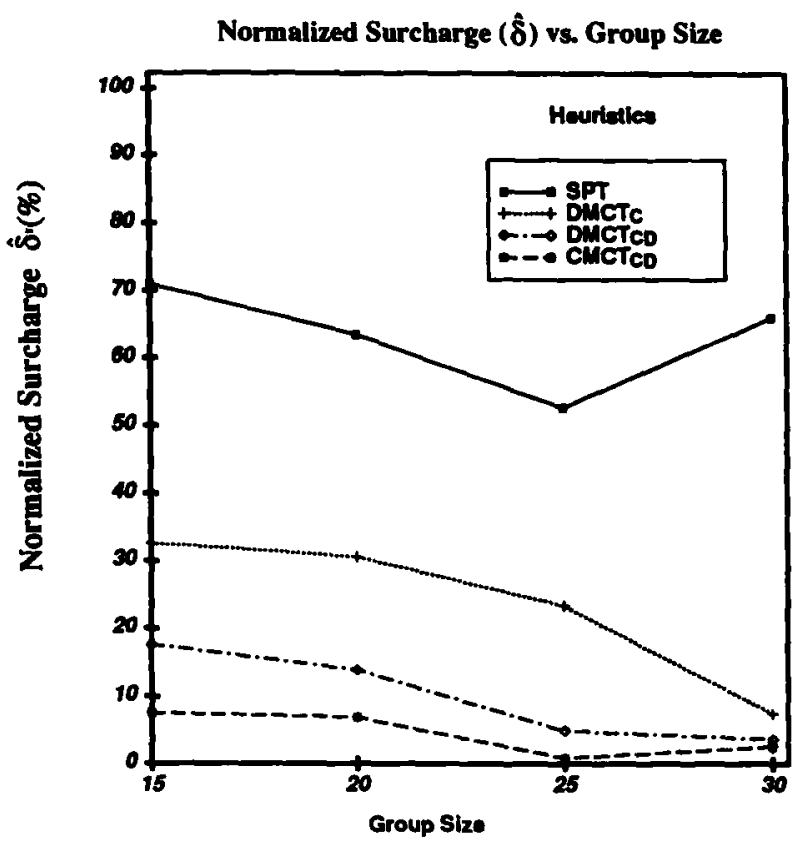

Fig. 10. Normalized surcharge $\hat{\delta}$ vs. group size, for number of nodes $=60$, and $\Delta=80$, with average degree of each node $=5$, when compared with $C M C T_{C}$.

Normalized Surcharge $(\hat{\delta})$ vs. Delay Tolerance

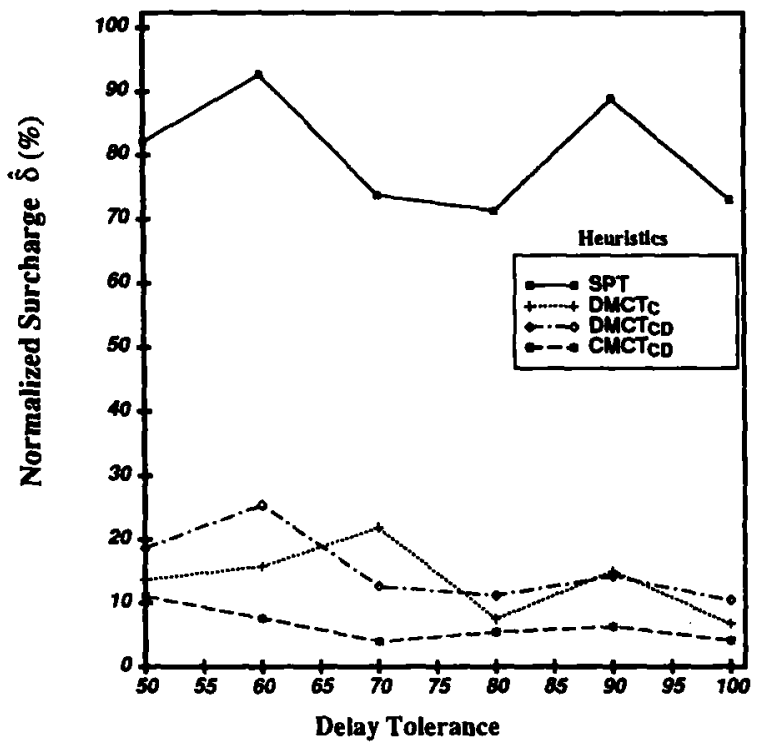

Fig. 11. Normalized surcharge $\hat{\delta}$ vs. delay tolerance for number of nodes $=50$, and group size $=$ 20 , with average degree of each node $=10$, when compared with $C M C T_{C}$. 
The problem of optimal multicast routing as formulated is intractable. However, we have shown that the distributed algorithms introduced produce extremely good solutions. They can scale to large sized graphs and still provide low cost routes with path delays satisfying the given tolerance. Comparing the performance of the distributed algorithms against the centralized approach we note that they come to within 5-30\%. Typical values for the normalized surcharge, $\hat{\delta}_{H}$, for the best distributed algorithm, $D M C T_{C D}$, are around $10 \%$ (compared to the best centralized heuristic $\left.C M C T_{C}\right)$.

Furthermore, we demonstrate that the solutions produced by the shortest delay algorithm are inferior to the solutions of the distributed algorithms with respect to cost. We also posit that, in selecting edges for inclusion in the constrained Steiner tree, it is advantageous to factor in the delay that is incurred on a path from the source through that edge. Our earlier results indicate that the same is not true for the corresponding centralized algorithms.

\section{ACKNOWLEDGMENTS}

The authors would like to thank the reviewers for their comments and especially for pointing out a flaw in a previous version of the cycle make-and-break procedure.

\section{APPENDIX A: THE MAIN PORTION OF THE DISTRIBUTED ALGORITHMS}

1. From the source $s$, send a FIND message up the tree formed so far to each node $v$ to determine the best out-going edge.

2. At node $v$, the best out-going edge is an edge determined by:

a. sending a TEST message on all adjacent edges $(v, w)$

b. asking each adjacent node $w$ which unreached destination nodes $d$ $\in S^{\prime}$ are reachable, if they are to incur a path delay of $P(w)$ from the source to $w$ and $S D(w, d)$ from $w$ to $d$

c. for each edge $(v, w)$, if at least one destination is reachable, and $w$ is not in the tree, then mark that edge as Usable

d. if at least one destination is reachable, but $w$ is already in the tree, then mark that edge as Cycle

e. if no destination is reachable, mark that edge as Unusable

f. for each Usable edge, determine the desirability of that edge using some heuristic, $f(v, w)$. The edge with the minimum value of $f(v$, $w)$ is the best out-going edge

3. Propagate the best out-going edge back down the tree towards the source, replacing it by better choices (i.e., with smaller values of $f(v$, w)) at other nodes along the way. 
4. At the source $s$

a. choose the best edge $(x, y)$, and add it to the tree by sending an ADD message up the branch that contains it

b. if $y$ is a destination node, remove it from $S^{\prime}$, the set of unreached destinations

5. At the node $x$, the node to which the new edge $(x, y)$ is to be added, mark the edge $(x, y)$ as Tree

6. At the new node $y$ just added to the tree

a. mark $y$ as being in the tree

b. mark edge $(y, x)$ as Reverse to denote that it is the first edge in the return path to $s$ from $y$

c. send an ADJUST message along each adjacent edge (except along ( $y$, $x)$ ) notifying the endpoints that if they are already in the tree, this edge should be marked as a Cycle edge

d. compute the path delay $P(y)$ from $s$ to $y$ to be the path delay from $s$ to $x$ plus the link delay on $(x, y)$, i.e., $P(y)=P(x)+D(x, y)$

7. If a node $v$ receives an ADJUST message to mark an edge as a Cycle edge

a. if that edge is already known to be Unusable, do not change its status

b. otherwise that edge must already be Usable, so change its status to Cycle

8. Repeat this process until all the destinations have been covered.

9. Prune the tree of unnecessary edges.

\section{APPENDIX B: THE CYCLE MAKE-AND-BREAK PHASE}

$$
\begin{gathered}
s=\text { source node } \\
x=\text { one endpoint of the Cycle edge } \\
y=\text { the other endpoint of the Cycle edge } \\
u, v, w=\text { arbitrary nodes in the cycle }
\end{gathered}
$$

6.1 At $s$, if no best out-going edge is passed down, then send a CYCLEMAKE message up the tree.

6.2 At node $v$, when a CYCLE-MAKE message is received, forward it up the tree, look at each adjacent Cycle edge $(v, w)$ that still leads to an unreached destination, and pick the most desirable of them, according to the selection function $f(v, w)$

6.3 The best out-going cycle-making edges are passed down the tree to the source $s$. 
6.4 At the source, send a CYCLE-ADD message up the tree with the edge that is determined to be the best.

6.5 At node $x$, if a CYCLE-ADD message is received with edge $(x, y)$, mark edge $(x, y)$ as Tree.

6.6 At node $x$, send a SEARCH-ANCESTOR-REVERSE message along its Reverse edge to determine the common ancestor of $x$ and $y$.

6.7 At node $y$, send a SEARCH-ANCESTOR-FORWARD on its Reverse path to determine the common ancestor of $x$ and $y$.

6.8 At node $w$, if a SEARCH-ANCESTOR-REVERSE message is received, pass it down its Reverse edge.

6.9 At node $w$, if a SEARCH-ANCESTOR-FORWARD message is received, pass it down its Reverse edge.

6.10 At node $w$, if both a SEARCH-ANCESTOR-REVERSE and a SEARCHANCESTOR-FORWARD message have been received, $w$ is the common ancestor of $x$ and $y$. Send a CYCLE-START message for node $x$ along the edge from which the SEARCH-ANCESTOR-REVERSE message came from.

6.11 At node $w$, if a CYCLE-START message is received, forward it along the edge on which the SEARCH-ANCESTOR-REVERSE message was received.

6.12 At node $x$, if a CYCLE-START message is received, create the cycle by sending a CYCLE-INCLUDE-EDGE message to $y$.

6.13 At node $y$, if a CYCLE-INCLUDE-EDGE message is received/

a. send a CYCLE-BREAK message down its Reverse edge

b. reset the status of the Reverse edge to Tree

c. reset the path delay to $P(x)+\mathscr{D}(x, y)$

d. mark edge $(y, x)$ as Reverse

6.14 At node $v$, if a CYCLE-BREAK message is received from node $u$, then switch the direction of the path by flipping the status to Reverse according to the conditions:

a. compute $N e w P(v)=P(u)+D(u, v)$

b. if $N e w P(v)<P(v)$ then

i. set the path delay at $v=\operatorname{NewP}(v)$

ii. let $(v, w)$ be the Reverse edge at $v$

iii. send a CYCLE-BREAK message to $w$

iv. set status of edge $(v, w)$ to Tree

$v$. set status of edge $(v, u)$ to Reverse

c. else

i. set status of edge $(v, u)$ to Unknown

ii. send CYCLE-STOP to node $u$

6.15 At node $u$, if a CYCLE-STOP is received from node $v$, set the status 
of edge $(u, v)$ to Unknown. Edge $(u, v)$ is the edge that is removed to break the cycle.

\section{REFERENCES}

1. S. Sabri and B. Prasada, Video conferencing systems, Proc. of the IEEE, Vol. 73, No. 4, pp. 671-688, April 1985.

2. S. Casner, K. Seo, W. Edmond, and C. Topolcic, $N$-way conferencing with packet video, Third International Workshop on Packet Video, March 1990.

3. W. D. Sincoskie, System architecture for a large scale video on demand service, Computer Networks and ISDN Systems, North-Holland, Vol. 22, No. 2, pp. 155-162, 1991.

4. J. Sutherland and L. Litteral, Residential Video Services, IEEE Comm. Mag., Vol. 30, No. 7, pp. 36-41, July 1992 .

5. B. Leiner, Ed., Critical issues in high-speed networking, RFC1077, Network Information Center, SRI International, Menlo Park, California, November 1988.

6. C. Partridge, Workshop Report, Internet research steering group workshop on very-high-speed networks, RFC1152, Network Information Center, SRI International, Menlo Park, California, April 1990.

7. AT\&T Bell Laboratories, Engineering and Operations in the Bell System, 1984.

8. D. Ferrari, Client requirements for real-time communication services, IEEE Communications Magazine, Vol. 28, No. 11, pp. 65-72, November 1990.

9. D. C. Verma, H. Zhang, and D. Ferrari, Delay jitter control for real-time communication in a packet switching network, Proc. TriComm '91, pp. 35-43, April 1991.

10. A. J. Frank, L. D. Wittie, and A. J. Bernstein, Multicast communication on network computers, IEEE Software, Vol. 2, No. 3, pp. 49-61, 1985.

11. M. Ahamad, (ed.), Multicast Communication in Distributed Systems, IEEE Computer Society Press, Technology Series, 1990.

12. S. Deering and D. Cheriton, Multicast routing in Intemetworks and extended LANs, $A C M$ Trans. on Computer Systems, Vol. 8, No. 2, pp. 85-110, May 1990.

13. M. R. Macedonia and D. P. Brutzman, MBone provides audio and video across the Internet, IEEE Computer, Vol. 27, No. 4, pp. 30-36, April 1994.

14. L. Zhang, S. Deering, D. Estrin, S. Shenker, and D. Zappala, RSVP: A new resource ReSerVation protocol, IEEE Network, Vol. 7, No. 5, pp. 8-18, September 1993.

15. T. Ballardie, P. Francis, and J. Crowcroft, Core Based Trees (CBT): An architecture for scalable inter-domain multicast routing, Proc. ACM SIGCOMM'93, San Francisco, California, pp. 85-95, September 1993.

16. K. Y. Eng, M. G. Hluchyj, and Y. S. Yeh, Multicast and broadcast services in a knockout packet switch, Proc. IEEE INFOCOM'88, pp. 29-34, March 1988.

17. J. S. Tumer, Design of a broadcast packet switching network, IEEE Trans. on Comm., Vol. 36, No. 6, pp. 734-743, June 1988.

18. T. T. Lee, Nonblocking copy networks for multicast packet switching, IEEE Journal Selected Areas in Communications, Vol. 6, No. 9, pp. 1455-1467, December 1988.

19. D. C. Verma and P. M. Gopal, Routing reserved bandwidth multi-point connections, Proc. ACM SIGCOMM'93, San Francisco, California, pp. 96-105, September 1993.

20. W, Yen and I. Akyildiz, Hierarchical Multicast Routing Protocol, 9th IEEE Workshop on Computer Comm., Marathon, Florida, October 1994. 
21. E. W. Dijkstra, A note on two problems in connexion with graphs, Numerische Mathematik, Vol. 1, pp. 269-271, 1959.

22. S. L. Hakimi, Steiner's problem in graphs and its implications, Networks, Vol. 1, pp. 113133, 1971.

23. R. M. Karp, Reducibility among combinatorial problems, In R. E. Miller and J. W. Thatcher, (eds.), Complexity of Computer Computations, pp. 85-103, Plenum Press, New York, 1972.

24. C.-H. Chow, On multicast path finding algorithms, Proc. IEEE INFOCOM'91, New York, pp. 1274-1283, 1991.

25. B. K. Kadaba and J. M. Jaffe, Routing to multiple destinations in computer networks, IEEE Trans. on Comm., Vol. 31, No. 3, pp. 343-351, March 1983.

26. D. W. Wall, Mechanisms for broadcast and selective broadcast, Ph.D. Thesis, Electrical Engineering Department, Stanford University, June 1980.

27. B. M. Waxman, Routing of multipoint connections, IEEE J. on Selected Areas in Comm., Vol. 6, No. 9, pp. 1617-1622, December 1988.

28. L. Kou, G. Markowsky, and L. Berman, A fast algorithm for Steiner trees, Acta Informatica, Vol. 15, pp. 141-145, 1981.

29. V. J. Rayward-Smith, The computation of nearly minimal Steiner trees in graphs, Intl. $J$. Math. Educ. Sci. Tech., Vol. 14, No. 1, pp. 15-23, 1983.

30. H. Takahashi and A. Matsuyama, An approximate solution for the Steiner problem in graphs, Math. Japonica, Vol. 6, pp. 573-577, 1980.

31. V. P. Kompella, J. C. Pasquale, and G. C. Polyzos, Multicasting for multimedia applications, Proc. IEEE INFOCOM'92, Florence, Italy, pp. 2078-2085, May 1992.

32. V. P. Kompella, J. C. Pasquale, and G. C. Polyzos, Multicast routing for multimedia communication, IEEE/ACM Trans. on Networking, Vol. 1, No. 3, pp. 286-292, June 1993.

33. R. E. Bellman, Dynamic Programming, Princeton University Press, Princeton, New Jersey, 1957.

34. R. G. Gallager, P. A. Humblett, and P. M. Spira, A distributed algorithm for minimum-weight spanning trees, ACM Trans. on Prog. Lang. Syst., Vol. 5, pp. 66-77, 1983.

35. L. Aguilar, J. J. Garcia-Luna-Aceves, D. Moran, E. J. Craighill, and R. Brungardt, Architecture for a multimedia teleconferencing system, Proc. ACM SIGCOMM'86, pp. 126-136, August 1986.

Vachaspathi P. Kompella received the Bachelor of Technology degree from the Indian Institute of Technology, Kanpur, India, and the M.S. and Ph.D. degrees from the University of California, San Diego. He joined IBM's Networking Systems Laboratory in Research Triangle Park, North Carolina, where he is working in the Network Studies department. His research interests are in the area of network support for multimedia, in resource reservation algorithms, quality of service specification and support, and multicast routing and delivery.

Joseph C. Pasquale is an associate professor in the Department of Computer Science and Engineering at the University of Califomia at San Diego. He received the B.S. and M.S. degrees from the Massachusetts Institute of Technology in 1982, and the Ph.D. degree from the University of California at Berkeley in 1988, all in computer science. He does research on network and operating system software design, especially to support $\mathrm{I} / \mathrm{O}$-intensive applications such as distributed multimedia (digital video and audio) and scientific computing. He also investigates issues of coordination and decentralized control in large distributed systems. Dr. Pasquale received the NSF Presidential Young Investigator Award in 1989. During 1991-94, Dr. Pasquale led a team of 
researchers to build the Sequoia 2000 Network, a wide-area high-speed network connecting five University of California campuses.

George C. Polyzos received the Diploma in Electrical Engineering from the National Technical University, Athens, Greece, and the M.A.Sc. in Electrical Engineering and the Ph.D. in Computer Science from the University of Toronto. He joined the faculty of the University of Califomia, San Diego, in September 1988, where he is now an Associate Professor at the Department of Computer Science and Engineering and co-director of the Computer Systems Laboratory. His research interests include communication network design and computer and communications systems performance evaluation. He has published papers in the areas of random access protocols, local and metropolitan area networks, supercomputer $1 / O$ characterization, Intemet traffic and performance characterization, and network and operating system support for multipoint multimedia communications. He is a member of the ACM, the IEEE and a Senior Fellow of the San Diego Supercomputer Center. 